\title{
SIMULATION OF ADAPTIVE CONTROL OF A TUBULAR CHEMICAL REACTOR
}

\author{
Petr Dostál, Jiří Vojtěšek, and Vladimír Bobál \\ Tomas Bata University in Zlin \\ Department of Process Control, Centre of Polymer Systems \\ nam. T.G. Masaryka 5555, \\ 76001 Zlin, Czech Republic \\ \{dostalp, bobal, vojtesek, babik\}@fai.utb.cz
}

\section{KEYWORDS}

Tubular chemical reactor, Nonlinear model, External linear model, Parameter identification, Polynomial approach, Pole assignment.

\begin{abstract}
The paper deals with continuous-time adaptive control of a tubular chemical reactor with the countercurrent cooling as a nonlinear single input - single output process. The nonlinear model of the reactor is approximated by an external linear model with parameters estimated via corresponding delta model. The control system structure with two feedback controllers is considered. The resulting controllers are derived using the polynomial approach. The method is tested on a mathematical model of the tubular chemical reactor.
\end{abstract}

\section{INTRODUCTION}

Tubular chemical reactor are units frequently used in chemical industry. From the system theory point of view, tubular chemical reactors belong to a class of nonlinear distributed parameter systems with mathematical models described by sets of nonlinear partial differential equations (NPDRs). The methods of modelling and simulation of such processes are described e.g. in (Luyben 1989), (Ingham et al. 1994) and (Dostál et al. 2008).

It is well known that the control of chemical reactors, and, tubular reactors especially, often represents very complex problem. The control problems are due to the process nonlinearity, its distributed nature, and high sensitivity of the state and output variables to input changes. Evidently, the process with such properties is hardly controllable by conventional control methods, and, its effective control requires application some of advanced methods.

One possible method to cope with this problem is using adaptive strategies based on an appropriate choice of a continuous-time external linear model (CT ELM) with recursively estimated parameters. These parameters are consequently used for parallel updating of controller's parameters. Some results obtained in this field were presented by authors of this paper e.g. in (Dostál et al. 2004).

For the CT ELM parameter estimation, either the direct method or application of an external delta model with the same structure as the CT model can be used, e.g. (Middleton and Goodwin 1990) or (Mukhopadhyay et al. 1992). Although delta models belong into discrete models, they do not have such disadvantageous properties connected with shortening of a sampling period as discrete $z$-models. In addition, parameters of delta models can directly be estimated from sampled signals. Moreover, it can be easily proved that these parameters converge to parameters of CT models for a sufficiently small sampling period (compared to the dynamics of the controlled process), as shown in (Stericker and Sinha 1993).

This paper deals with continuous-time adaptive control of a tubular chemical reactor with a countercurrent cooling. With respect to practical possibilities of a measurement and control, the mean reactant temperature temperature is chosen as the controlled output, and, the coolant flow rate as the control input. The nonlinear model of the reactor is approximated by a CT external linear model with a structure chosen on the basis of computed controlled output step responses. The control structure with two feedback controllers is considered, e.g. (Dostál et al. 2007). The resulting controllers are derived using the polynomial approach (Kučera 1993) and the pole assignment method, e.g. (Bobál et al. 2005). The method is tested on a mathematical model of a tubular chemical reactor.

\section{MODEL OF THE REACTOR}

An ideal plug-flow tubular chemical reactor with a simple exothermic consecutive reaction $A \stackrel{k_{1}}{\rightarrow} B \stackrel{k_{2}}{\rightarrow} C$ in the liquid phase and with the countercurrent cooling is considered. Heat losses and heat conduction along the metal walls of tubes are assumed to be negligible, but dynamics of the metal walls of tubes are significant. All densities, heat capacities, and heat transfer coefficients are assumed to be constant. Under above assumptions, the reactor model can be described by five PDRs in the form

$$
\begin{gathered}
\frac{\partial c_{A}}{\partial t}+v_{r} \frac{\partial c_{A}}{\partial z}=-k_{1} c_{A} \\
\frac{\partial c_{B}}{\partial t}+v_{r} \frac{\partial c_{B}}{\partial z}=k_{1} c_{A}-k_{2} c_{B} \\
\frac{\partial T_{r}}{\partial t}+v_{r} \frac{\partial T_{r}}{\partial z}=\frac{Q_{r}}{\left(\rho c_{p}\right)_{r}}-\frac{4 U_{1}}{d_{1}\left(\rho c_{p}\right)_{r}}\left(T_{r}-T_{w}\right)
\end{gathered}
$$




$$
\begin{aligned}
& \frac{\partial T_{w}}{\partial t}=\frac{4}{\left(d_{2}^{2}-d_{1}^{2}\right)\left(\rho c_{p}\right)_{w}}\left[d_{1} U_{1}\left(T_{r}-T_{w}\right)+\right. \\
&+d_{2} U_{2}\left(T_{c}-T_{w}\right] \\
& \frac{\partial T_{c}}{\partial t}-v_{c} \frac{\partial T_{c}}{\partial z}=\frac{4 n_{1} d_{2} U_{2}}{\left(d_{3}^{2}-n_{1} d_{2}^{2}\right)\left(\rho c_{p}\right)_{c}}\left(T_{w}-T_{c}\right)
\end{aligned}
$$

with initial conditions

$c_{A}(z, 0)=c_{A}^{s}(z), \quad c_{B}(z, 0)=c_{B}^{s}(z), \quad T_{r}(z, 0)=T_{r}^{S}(z)$,

$T_{w}(z, 0)=T_{w}^{s}(z), T_{c}(z, 0)=T_{c}^{s}(z)$

and boundary conditions

$c_{A}(0, t)=c_{A 0}(t)\left(\mathrm{kmol} / \mathrm{m}^{3}\right)$,

$c_{B}(0, t)=c_{B 0}(t)\left(\mathrm{kmol} / \mathrm{m}^{3}\right), T_{r}(0, t)=T_{r 0}(t)(\mathrm{K})$,

$T_{c}(L, t)=T_{c L}(t)(\mathrm{K})$.

Here, $t$ is the time, $z$ is the axial space variable, $c$ are concentrations, $T$ are temperatures, $v$ are fluid velocities, $d$ are diameters, $\rho$ are densities, $c_{p}$ are specific heat capacities, $U$ are heat transfer coefficients, $n_{1}$ is the number of tubes and $L$ is the length of tubes. The subscript $(\cdot)_{r}$ stands for the reactant mixture, $(\cdot)_{w}$ for the metal walls of tubes, $(\cdot)_{c}$ for the coolant, and the superscript $(\cdot)^{s}$ for steady-state values.

The reaction rates and heat of reactions are nonlinear functions expressed as

$$
\begin{gathered}
k_{j}=k_{j 0} \exp \left(\frac{-E_{j}}{R T_{r}}\right), j=1,2 \\
Q_{r}=\left(-\Delta H_{r 1}\right) k_{1} c_{A}+\left(-\Delta H_{r 2}\right) k_{2} c_{B}
\end{gathered}
$$

where $k_{0}$ are pre-exponential factors, $E$ are activation energies, $\left(-\Delta H_{r}\right)$ are in the negative considered reaction entalpies, and $R$ is the gas constant.

The fluid velocities are calculated via the reactant and coolant flow rates as

$$
v_{r}=\frac{4 q_{r}}{\pi n_{1} d_{1}^{2}}, v_{c}=\frac{4 q_{c}}{\pi\left(d_{3}^{2}-n_{1} d_{2}^{2}\right)}
$$

The parameter values with correspondent units used for simulations are given in Table 1.

Table 1. Used parameter values

\begin{tabular}{|l|l|}
\hline$L=8 \mathrm{~m}$ & $n_{1}=1200$ \\
\hline$d_{1}=0.02 \mathrm{~m}$ & $d_{2}=0.024 \mathrm{~m}$ \\
\hline \multicolumn{2}{|c|}{$d_{3}=1 \mathrm{~m}$} \\
\hline$\rho_{r}=985 \mathrm{~kg} / \mathrm{m}^{3}$ & $c_{p r}=4.05 \mathrm{~kJ} / \mathrm{kg} \mathrm{K}$ \\
\hline$\rho_{w}=7800 \mathrm{~kg} / \mathrm{m}^{3}$ & $c_{p w}=0.71 \mathrm{~kJ} / \mathrm{kg} \mathrm{K}$ \\
\hline$\rho_{c}=998 \mathrm{~kg} / \mathrm{m}^{3}$ & $c_{p c}=4.18 \mathrm{~kJ} / \mathrm{kg} \mathrm{K}$ \\
\hline$U_{1}=2.8 \mathrm{~kJ} / \mathrm{m}^{2} \mathrm{~S} \mathrm{~K}$ & $U_{2}=2.56 \mathrm{~kJ} / \mathrm{m}^{2} \mathrm{~s} \mathrm{~K}$ \\
\hline$k_{10}=5.61 \cdot 10^{16} 1 / \mathrm{s}$ & $k_{20}=1.128 \cdot 10^{18} 1 / \mathrm{s}$ \\
\hline$E_{1} / R=13477 \mathrm{~K}$ & $E_{2} / R=15290 \mathrm{~K}$ \\
\hline$\left(-\Delta H_{r 1}\right)=5.8 \cdot 10^{4} \mathrm{~kJ} / \mathrm{kmol}$ & $\left(-\Delta H_{r 2}\right)=1.8 \cdot 10^{4} \mathrm{~kJ} / \mathrm{kmol}$ \\
\hline
\end{tabular}

From the system engineering point of view, $c_{A}(L, t)=c_{A \text { out }}, \quad c_{B}(L, t)=c_{B \text { out }}, \quad T_{r}(L, t)=T_{\text {rout }}$ and $T_{c}(0, t)=T_{c \text { out }}$ are the output variables, and, $q_{r}(t)$, $q_{c}(t), c_{A 0}(t), T_{r 0}(t)$ and $T_{c L}(t)$ are the input variables. Among them, for the control purposes, mostly the coolant flow rate can be taken into account as the control variable, whereas other inputs entering into the process can be accepted as disturbances. In this paper, the mean reactant temperature given by

$$
T_{m}(t)=\frac{1}{L} \int_{0}^{L} T_{r}(z, t) d z
$$

is considered as the controlled output.

\section{COMPUTATION MODELS}

For computation both steady-state and dynamic characteristics, the finite differences method is employed. The procedure is based on substitution of the space interval $z \in<0, L\rangle$ by a set of discrete node points $\left\{z_{i}\right\}$ for $i=1, \ldots, n$, and, subsequently, by approximation of derivatives with respect to the space variable in each node point by finite differences.

\section{Dynamic Model}

Using the finite differences method, nonlinear PDEs (1) - (5) are approximated by a set of nonlinear ODEs in the form

$$
\begin{aligned}
& \frac{d c_{A}(i)}{d t}=-\left[b_{0}+k_{1}(i)\right] c_{A}(i)+b_{0} c_{A}(i-1) \\
& \frac{d c_{B}(i)}{d t}=k_{1}(i) c_{A}(i)-\left[b_{0}+k_{2}(i)\right] c_{B}(i)+ \\
& +b_{0} c_{B}(i-1) \\
& \frac{d T_{r}(i)}{d t}=b_{1} Q_{r}(i)-\left(b_{0}+b_{2}\right) T_{r}(i)+b_{0} T_{r}(i-1)+ \\
& +b_{2} T_{w}(i) \\
& \frac{d T_{w}(i)}{d t}=b_{3}\left[T_{r}(i)-T_{w}(i)\right]+b_{4}\left[T_{c}(i)-T_{w}(i)\right] \\
& \frac{d T_{c}(m)}{d t}=-\left(b_{5}+b_{6}\right) T_{c}(m)+b_{5} T_{c}(m+1)+ \\
& +b_{6} T_{w}(m)
\end{aligned}
$$

for $i=1, \ldots, n$ and $m=n-i+1$, and, with initial conditions

$$
\begin{aligned}
& c_{A}(i, 0)=c_{A}^{s}(i), \quad c_{B}(i, 0)=c_{B}^{s}(i), \quad T_{r}(i, 0)=T_{r}^{s}(i), \\
& T_{w}(i, 0)=T_{w}^{s}(i) \text { and } T_{c}(i, 0)=T_{c}^{s}(i) \text { for } i=1, \ldots, n .
\end{aligned}
$$

The boundary conditions enter into Eqs. (10) - (12) and (14) for $i=1$.

Now, nonlinear functions in Eqs. (10) - (14) take the discrete form 


$$
\begin{gathered}
k_{j}(i)=k_{j 0} \exp \left(\frac{-E_{j}}{R T_{r}(i)}\right), j=1,2 \\
Q_{r}(i)=\left(-\Delta H_{r 1}\right) k_{1}(i) c_{A}(i)+ \\
+\left(-\Delta H_{r 2}\right) k_{2}(i) c_{B}(i)
\end{gathered}
$$

for $i=1, \ldots, n$.

The parameters $b$ in Eqs. (10) - (14) are calculated from formulas

$$
\begin{gathered}
b_{0}=\frac{v_{r}}{h}, b_{1}=\frac{1}{\left(\rho c_{p}\right)_{r}}, b_{2}=\frac{4 U_{1}}{d_{1}\left(\rho c_{p}\right)_{r}}, \\
b_{3}=\frac{4 d_{1} U_{1}}{\left(d_{2}^{2}-d_{1}^{2}\right)\left(\rho c_{p}\right)_{w}}, b_{4}=\frac{4 d_{2} U_{2}}{\left(d_{2}^{2}-d_{1}^{2}\right)\left(\rho c_{p}\right)_{w}} \\
b_{5}=\frac{v_{c}}{h}, b_{6}=\frac{4 n_{1} d_{2} U_{2}}{\left(d_{3}^{2}-n_{1} d_{2}^{2}\right)\left(\rho c_{p}\right)_{c}} .
\end{gathered}
$$

Here, the formula for computation of $T_{m}$ takes the discrete form

$$
T_{m}(t)=\frac{1}{n} \sum_{i=1}^{n} T_{r}\left(z_{i}, t\right)
$$

\section{Steady-state Model}

Computation of the steady-state characteristics is necessary not only for a steady-state analysis but the steady state values $y^{s}(i)$ also constitute initial conditions in ODRs (10) - (14) (here, $y$ presents some of the variable in the set $(10)-(14))$.

The steady-state model can simply be derived equating the time derivatives in $(10)-(14)$ to zero.

\section{Steady-state and Dynamic Characteristics}

Typical reactant temperature profiles along the reactor tubes computed for $c_{A 0}^{s}=2.85, c_{B 0}^{s}=0, T_{r 0}^{s}=323$, $T_{c 0}^{s}=293$ and $q_{r}^{s}=0.15$ for various coolant flow rates are shown in Fig. 1. A presence of a maximum on the reactant temperature profiles is a common property of many tubular reactors with exothermic reactions.

A dependence of the reactant mean temperature on the coolant flow rate is shown in Fig. 2. The form of the curve documents a nonlinear relation between supposed controlled output and the coolant flow rate which is considered as the control input.

Dynamic charakteristics were computed in the neighbourhood of the chosen operating point $q_{c}^{s}=0.27 \mathrm{~m}^{3} / \mathrm{s}, \quad T_{m}^{s}=334.44 \mathrm{~K}$. For the dynamic analysis and subsequent control purposes, the controlled output is defined as a deviation from the steady value

$$
y(t)=\Delta T_{m}(t)=T_{m}(t)-T_{m}^{s} .
$$

Such form is frequently used in the control. The deviation of the coolant flow rate is denoted as

$$
\Delta q_{c}=q_{c}(t)-q_{c}^{s} .
$$

The responses of the output to the coolant flow rate step changes are shown in Fig. 3.

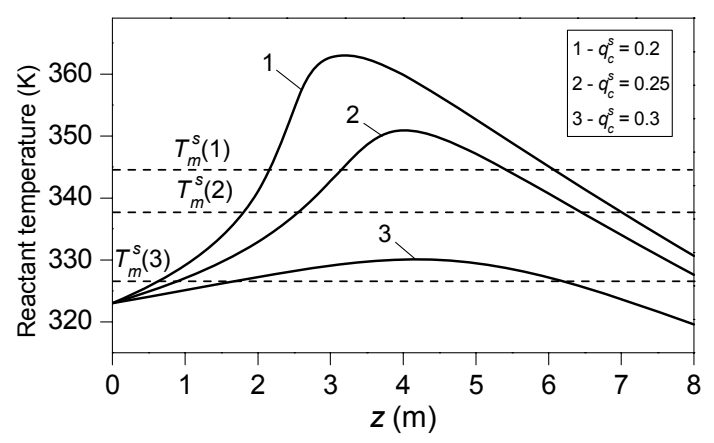

Fig. 1. Reactant temperature profiles for various coolant flow rates.

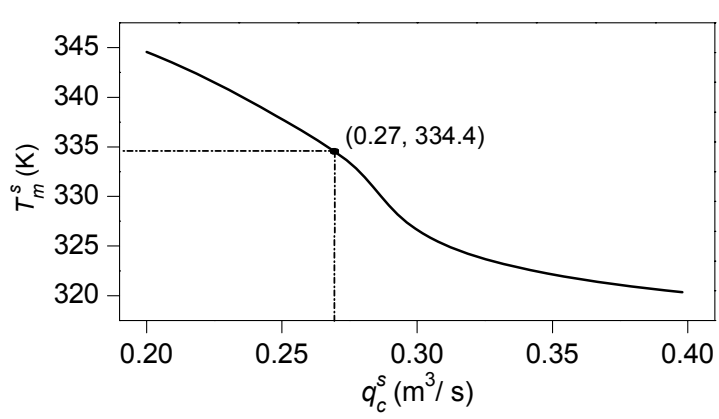

Fig. 2. Dependence of the reactant mean temperature on the coolant flow rates.

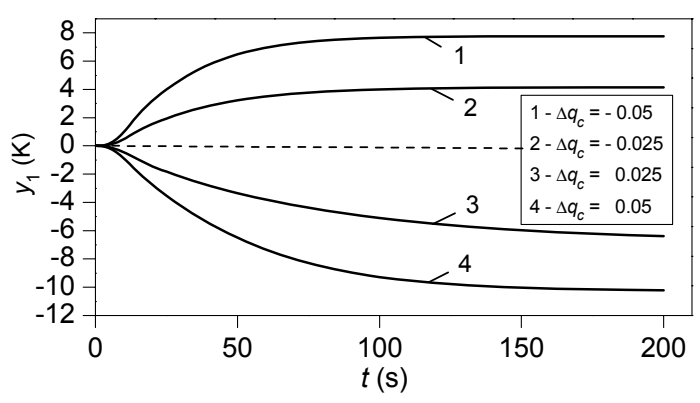

Fig. 3. Reactant mean temperature step responses.

The above shown responses document nonlinear behaviour of the reactant mean temperature.

\section{CT AND DELTA ELM}

For the control purposes, the control input variable are considered in the form

$$
u(t)=10 \frac{q_{c}(t)-q_{c}^{s}}{q_{c}^{s}}
$$

This expression enables to obtain control input and controlled output variables of approximately the same magnitude.

A choice of the CT ELM structure does not stem from known structure of the model (1) - (5) but from a character of simulated step responses. It is well known that in adaptive control a controlled process of a higher 
order can be approximated by a linear model of a lower order with variable parameters. Taking into account profiles of curves in Fig. 3 with zero derivatives in $t=0$, the second order CT ELM has been chosen in the form of the second order linear differential equation

$$
\ddot{y}(t)+a_{1} \dot{y}(t)+a_{0} y(t)=b_{0} u(t)
$$

and, in the complex domain, as the transfer function

$$
G(s)=\frac{b_{0}}{s^{2}+a_{1} s+a_{0}} .
$$

Establishing the $\delta$ operator

$$
\delta=\frac{q-1}{T_{0}}
$$

where $q$ is the forward shift operator and $T_{0}$ is the sampling period, the delta ELM corresponding to (22) takes the form

$$
\delta^{2} y\left(t^{\prime}\right)+a_{1}^{\prime} \delta y\left(t^{\prime}\right)+a_{0}^{\prime} y\left(t^{\prime}\right)=b_{0}^{\prime} u\left(t^{\prime}\right)
$$

where $t^{\prime}$ is the discrete time. When the sampling period is shortened, the delta operator approaches the derivative operator, and, the estimated parameters $a^{\prime}, b^{\prime}$ reach the parameters $a, b$ of the CT model (22).

\section{DELTA MODEL PARAMETER ESTIMATION}

Substituting $t^{\prime}=k-2$, equation (25) can be rewriten to the form

$$
\delta^{2} y(k-2)+a_{1}^{\prime} \delta y(k-2)+a_{0}^{\prime} y(k-2)=b_{0}^{\prime} u(k-2)
$$

Establishing the regression vector

$$
\boldsymbol{\Phi}_{\delta}^{T}(k-1)=(-\delta y(k-2) \quad-y(k-2) \quad u(k-2))
$$

where

$$
\delta y(k-2)=\frac{y(k-1)-y(k-2)}{T_{0}}
$$

the vector of delta model parameters

$$
\boldsymbol{\Theta}_{\delta}^{T}(k)=\left(a_{1}^{\prime} a_{0}^{\prime} b_{0}^{\prime}\right)
$$

is recursively estimated using least squares method with exponential and directional forgetting (Bobál et al. 2005) from the ARX model

$$
\delta^{2} y(k-2)=\boldsymbol{\Theta}_{\delta}^{T}(k) \boldsymbol{\Phi}_{\delta}(k-1)+\varepsilon(k)
$$

where

$$
\delta^{2} y(k-2)=\frac{y(k)-2 y(k-1)+y(k-2)}{T_{0}^{2}}
$$

\section{CONTROLLER DESIGN}

The control system with two feedback controllers is depicted in Fig. 4.

In the scheme, $w$ is the reference signal, $v$ denotes the load disturbance, $e$ the tracking error, $u_{0}$ output of controllers, $u$ the control input and $y$ the controlled output. The transfer function $G(s)$ of the CT ELM is given by (23).

The reference $w$ and the disturbance $v$ are considered as

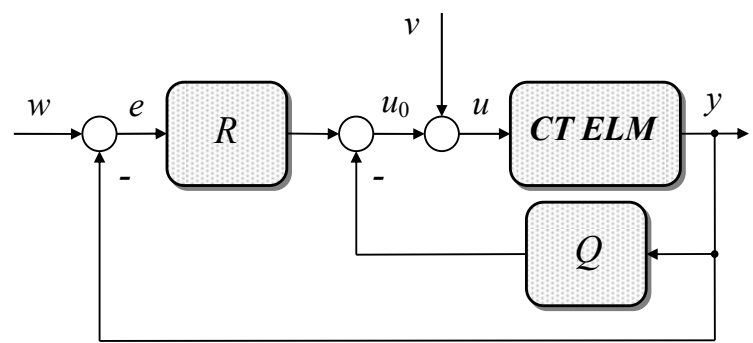

Fig. 4. Control system with two feedback controllers

the step functions with transforms

$$
W(s)=\frac{w_{0}}{s}, V(s)=\frac{v_{0}}{s} .
$$

The transfer functions of both controllers are in forms

$$
R(s)=\frac{r(s)}{\tilde{p}(s)}, \quad Q(s)=\frac{\tilde{q}(s)}{\tilde{p}(s)}
$$

where $\tilde{q}, r$ and $\tilde{p}$ are coprime polynomials in $s$ fulfilling the condition of properness $\operatorname{deg} r \leq \operatorname{deg} \tilde{p}$ and $\operatorname{deg} q \leq \operatorname{deg} \tilde{p}$.

The controller design described in this section appears from the polynomial approach. The general requirements on the control system are formulated as its internal properness and stability, asymptotic tracking of the reference and load disturbance attenuation. The procedure to derive admissible controllers can briefly be performed as follows:

Let the polynomial $t$ has the form

$$
t(s)=r(s)+\widetilde{q}(s) .
$$

Then, the control system stability is ensured when polynomials $\tilde{p}$ and $t$ are given by a solution of the polynomial equation

$$
a(s) \tilde{p}(s)+b(s) t(s)=d(s)
$$

with a stable polynomial $d$ on the right side. Evidently, the roots of $d$ determine the closed-loop poles.

Taking into account the transform of the tracking error

$$
E(s)=\frac{1}{d}[(a \tilde{p}+b \tilde{q}) W(s)-b \tilde{p} V(s)]
$$

and both transforms (32), the asymptotic tracking and load disturbance attenuation are provided by polynomials $\tilde{p}$ and $\tilde{q}$ having the form

$$
\tilde{p}(s)=s p(s), \tilde{q}(s)=s q(s) .
$$

Subsequently, the transfer functions (33) take forms

$$
Q(s)=\frac{q(s)}{p(s)}, R(s)=\frac{r(s)}{s p(s)}
$$

and, a stable polynomial $p(s)$ in their denominators ensures the stability of controllers.

Now, the polynomial $t$ can be rewritten to the form

$$
t(s)=r(s)+s q(s) .
$$

Taking into account the solvability of (35) and the 
condition of internal properness, the degrees of polynomials in (35) and (38) can be easily derived as

$$
\begin{gathered}
\operatorname{deg} t=\operatorname{deg} r=\operatorname{deg} a, \operatorname{deg} q=\operatorname{deg} a-1, \\
\operatorname{deg} p \geq \operatorname{deg} a-1, \operatorname{deg} d \geq 2 \operatorname{deg} a .
\end{gathered}
$$

Denoting $\operatorname{deg} a=n$, polynomials $t, r$ and $q$ have forms

$$
t(s)=\sum_{i=0}^{n} t_{i} s^{i}, r(s)=\sum_{i=0}^{n} r_{i} s^{i}, q(s)=\sum_{i=1}^{n} q_{i} s^{i-1}
$$

and, relations among their coefficients are

$$
r_{0}=t_{0}, \quad r_{i}+q_{i}=t_{i} \text { for } i=1, \ldots, n .
$$

Since by a solution of the polynomial equation (35) provides calculation of coefficients $t_{i}$, unknown coefficients $r_{i}$ and $q_{i}$ can be obtained by a choice of selectable coefficients $\beta_{i} \in\langle 0,1\rangle$ such that

$$
r_{i}=\beta_{i} t_{i}, \quad q_{i}=\left(1-\beta_{i}\right) t_{i} \text { for } i=1, \ldots, n .
$$

The coefficients $\beta_{i}$ distribute a weight between numerators of transfer functions $Q$ and $R$.

Remark: If $\beta_{i}=1$ for all $i$, the control system in Fig. 4 reduces to the $1 \mathrm{DOF}$ control configuration $(Q=0)$. If $\beta_{i}=0$ for all $i$, and, both reference and load disturbance are step functions, the control system corresponds to the 2DOF control configuration.

For the second order model (23) with $\operatorname{deg} a=2$, the controller's transfer functions take specific forms

$$
\begin{aligned}
& Q(s)=\frac{q(s)}{p(s)}=\frac{q_{2} s+q_{1}}{s+p_{0}} \\
& R(s)=\frac{r(s)}{s p(s)}=\frac{r_{2} s^{2}+r_{1} s+r_{0}}{s\left(s+p_{0}\right)}
\end{aligned}
$$

where

$$
\begin{gathered}
r_{0}=t_{0}, r_{1}=\beta_{1} t_{1}, r_{2}=\beta_{2} t_{2}, \\
q_{1}=\left(1-\beta_{1}\right) t_{1}, q_{2}=\left(1-\beta_{2}\right) t_{2} .
\end{gathered}
$$

The controller parameters then result from a solution of the polynomial equation (35) and depend upon coefficients of the polynomial $d$. The next problem here is to find a stable polynomial $d$ that enables to obtain acceptable stabilizing controllers.

In this paper, the polynomial $d$ with roots determining the closed-loop poles is chosen as

$$
d(s)=n(s)(s+\alpha)^{2}
$$

where $n$ is a stable polynomial obtained by spectral factorization

$$
a^{*}(s) a(s)=n^{*}(s) n(s)
$$

and $\alpha$ is the selectable parameter.

Note that a choice of $d$ in the form (46) provides the control of a good quality for aperiodic controlled processes.

The coefficients $n$ then are expressed as

$$
n_{0}=\sqrt{a_{0}^{2}}, n_{1}=\sqrt{a_{1}^{2}+2 n_{0}-2 a_{0}}
$$

and, the controller parameters $p_{0}$ and $t$ can be obtained from solution of the matrix equation

$$
\left(\begin{array}{cccc}
1 & 0 & 0 & 0 \\
a_{1} & b_{0} & 0 & 0 \\
a_{0} & 0 & b_{0} & 0 \\
0 & 0 & 0 & b_{0}
\end{array}\right) \times\left(\begin{array}{c}
p_{0} \\
t_{2} \\
t_{1} \\
t_{0}
\end{array}\right)=\left(\begin{array}{c}
d_{3}-a_{1} \\
d_{2}-a_{0} \\
d_{1} \\
d_{0}
\end{array}\right)
$$

where

$$
\begin{aligned}
& d_{3}=n_{1}+2 \alpha, d_{2}=2 \alpha n_{1}+n_{0}+\alpha^{2} \\
& d_{1}=2 \alpha n_{0}+\alpha^{2} n_{1}, d_{0}=\alpha^{2} n_{0}
\end{aligned}
$$

Now, it follows from the above introduced procedure that tuning of controllers can be performed by a suitable choice of selectable parameters $\beta$ and $\alpha$.

The controller parameters $r$ and $q$ can then be obtained from (45).

The adaptive control system is shown in Fig. 5.

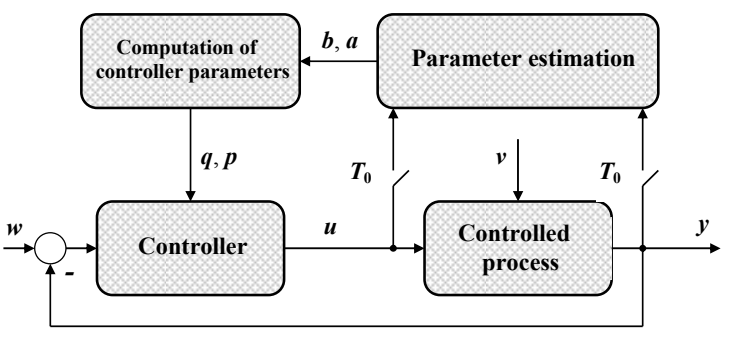

Fig. 5. Adaptive control scheme.

\section{CONTROL SIMULATION}

Also the control simulations were performed in a neighbourhood of the operating point $q_{c}^{s}=0.27 \mathrm{~m}^{3} / \mathrm{s}$, $T_{m}^{s}=334.44 \mathrm{~K}$, For the start (the adaptation phase), the $\mathrm{P}$ controller with a small gain was used in all simulations.

The effect of the pole $\alpha$ on the controlled output responses is transparent from Fig. 6. Here, two values of $\alpha$ were selected. The control simulation shows sensitivity of the controlled output to $\alpha$. The higher values of this parameter speed the control, however, they provide greater overshoots (undershoots). Other here not shown simulations demonstrated that a careless selection of the parameter $\alpha$ can lead to controlled output responses of a poor quality, to oscillations or even to the control instability. Moreover, an increasing $\alpha$ leads to higher values and changes of the control input as shown in Fig. 7. This fact can be important in control of real technological processes.

The controlled output $y$ response for two values $\beta_{2}$ is shown in Fig. 8. It can be seen that an effect of this parameterer is insignificant.

The controlled output responses documenting an effect of the parameter $\beta_{1}$ are in Fig. 9. There, a higher value of $\beta_{1}$ results in greater overshoots (undershoots) of the controlled output.

Corresponding control input responses can be seen in Fig. 10. It can be seen that an increasing $\beta_{1}$ leads to greater values of inputs. 


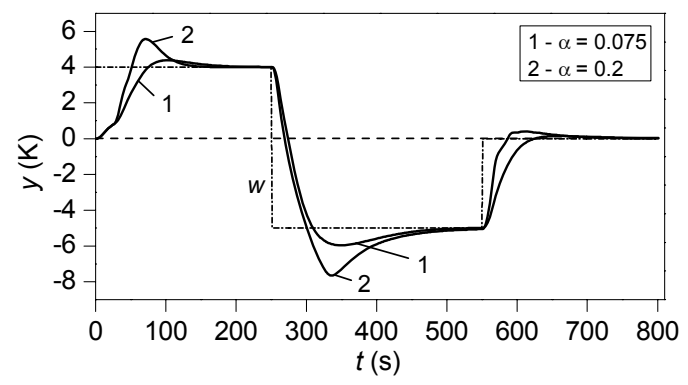

Fig. 6. Controlled output $y_{1}$ responses: effect of $\alpha$ $\left(\beta_{1}=1, \beta_{2}=0.5\right)$.

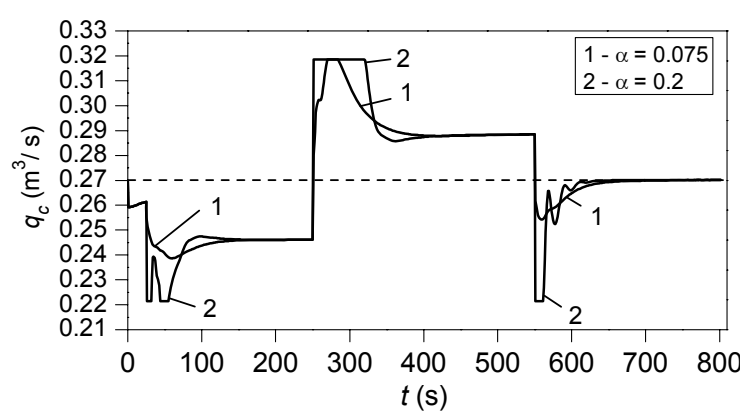

Fig. 7. Coolant flow rate responses in control of reactant mean temperature - effect of $\alpha$ $\left(\beta_{1}=1, \beta_{2}=0.5\right)$.

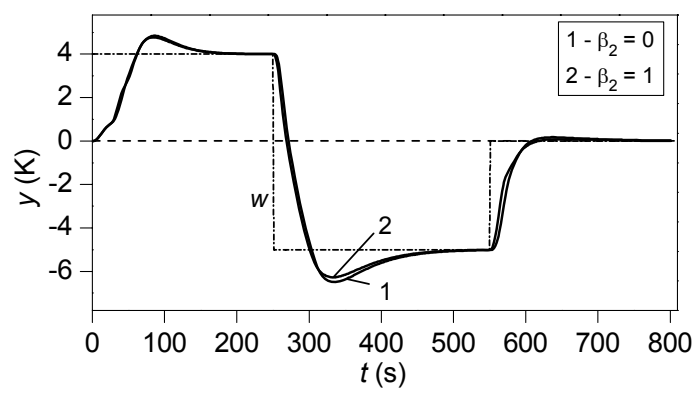

Fig. 8. Controlled output responses: effect of $\beta_{2}$ $\left(\alpha=0.1, \beta_{1}=1\right)$.

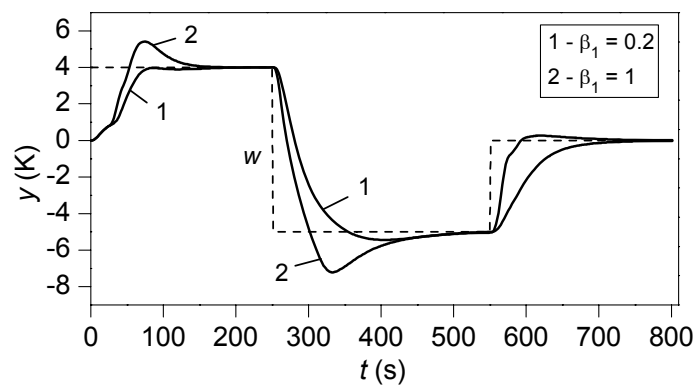

Fig. 9. Controlled output responses: effect of $\beta_{1}$ $\left(\alpha=0.15, \beta_{2}=0\right)$.

Of interest, the evolution of estimated CT ELM parameters in control of the reactant mean temperature is shown in Fig. 11.

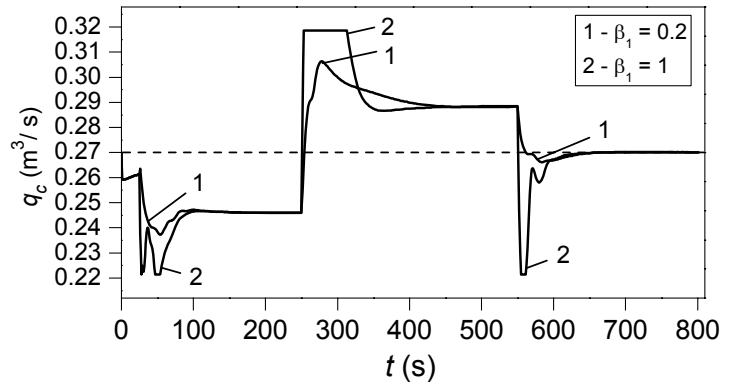

Fig. 10. Coolant flow rate responses in control of reactant mean temperature - effect of $\beta_{1}$ $\left(\alpha=0.15, \beta_{2}=0\right)$.

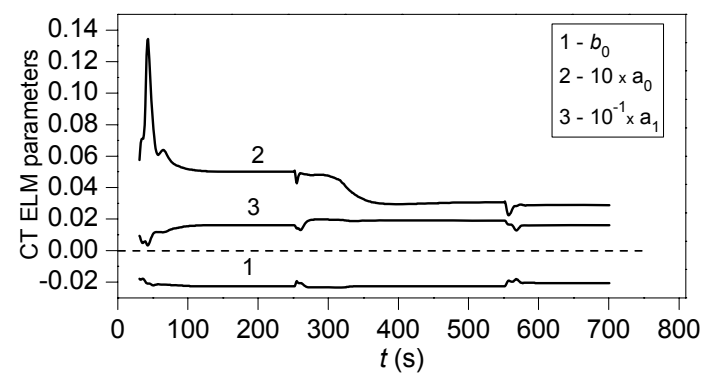

Fig. 11. CT ELM parameter evolution $(\alpha=0.15$, $\left.\beta_{1}=1, \beta_{2}=0\right)$.

A presence of an integrating part in the controller enables rejection of various step disturbances entering into the process. As an example, step disturbances attenuation for the output $y$ is presented. Step disturbances $\Delta c_{A 0}=0.15 \mathrm{kmol} / \mathrm{m}^{3}, \Delta q_{r}=-0.03 \mathrm{~m}^{3} / \mathrm{s}$ and $\Delta T_{r 0}=2 \mathrm{~K}$ were injected into the nonlinear model of the reactor in times $t_{v}=220 \mathrm{~s}, t_{v}=440 \mathrm{~s}$ and $t_{v}=640 \mathrm{~s}$. The controller parameters were estimated only in the first (tracking) interval $t<200 \mathrm{~s}$. The authors' experiences proved that an utilization of recursive identification using the delta model after reaching of a constant reference and in presence of step disturbances decreases the control quality. From this reason, during interval $t \geq 200 \mathrm{~s}$, fixed parameters were used. The controlled output responses $y$ are shown in Fig. 12.

To illustrate an effect of an additive random disturbance, the result of the controlled output $y$ simulation in a presence of the random signal $v(t)=c_{A 0}(t)-c_{A}^{s}$ is shown in Fig. 13.

\section{CONCLUSIONS}

In this paper, one approach to continuous-time adaptive control of the mean reactant temperatures in a tubular chemical reactor was proposed. The control strategy is based on the preliminary steady-state and dynamic analysis of the process and on the assumption of the temperature measurement along the reactor. The proposed algorithm employs an alternative continuoustime external linear model with parameters obtained 


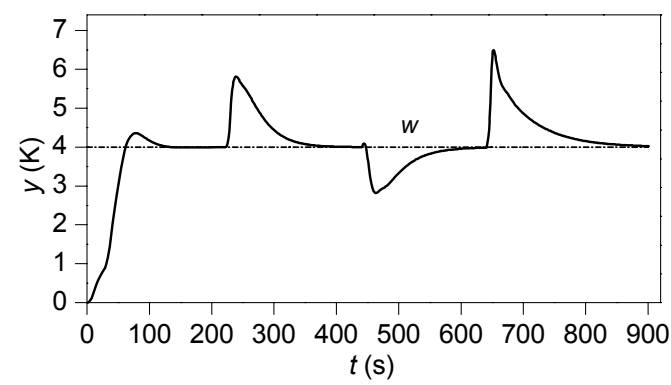

Fig. 12. Controlled output in presence of step disturbances $\left(\alpha=0.15, \beta_{1}=0.5, \beta_{2}=0\right)$.

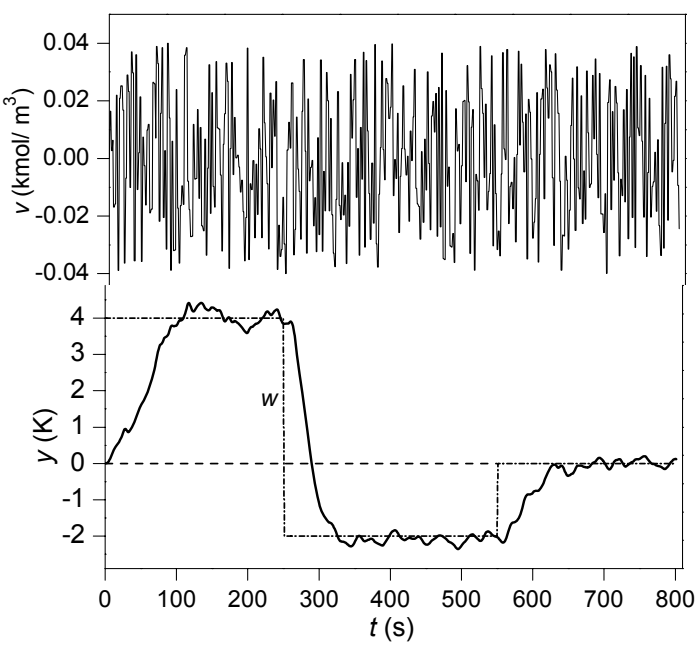

Fig. 13. Controlled output in the presence of random disturbance in $c_{A 0}(\alpha=0.15)$.

through recursive parameter estimation of a corresponding delta model. The control system structure with two feedback controllers is considered. Resulting continuous-time controllers are derived using the polynomial approach and given by a solution of the polynomial equation. Tuning of their parameters is possible via closed-loop pole assignment. The presented method has been tested by computer simulation on the nonlinear model of the tubular chemical reactor with a consecutive exothermic reaction. The simulation results demonstrate an applicability of the presented control strategy.

\section{ACKNOWLEDGMENT}

This article was created with support of Operational Programme Research and Development for Innovations co-funded by the European Regional Development Fund (ERDF) and national budget of Czech Republic within the framework of the Centre of Polymer Systems project (reg.number: CZ.1.05/2.1.00/03.0111).

\section{REFERENCES}

Luyben, W. 1989. Process modelling, simulation and control for chemical engineers. McGraw-Hill, New York.

Ingham, J., I.J. Dunn, E. Heinzle, and J. E. Přenosil. 1994. Chemical Engineering Dynamic: Modelling with PC Simulation. VCH Verlagsgesellschaft, Weinheim.

Dostál, P., V. Bobál, and J. Vojtěšek. 2008. "Simulation of steady-state and dynamic behaviour of a tubular chemical reactor". In Proc. 22nd European Conference on Modelling and Simulation, Nicosia, Cyprus, 487-492.

Dostál, P., V. Bobál and F. Gazdoš, F. 2004. "Adaptive control of nonlinear processes: Continuous-time versus delta model parameter estimation". In IFAC Workshop on Adaptation and Learning in Control and Signal Processing ALCOSP 04, Yokohama, Japan, 273-278.

Middleton, R.H. and G.C. Goodwin. 1990. Digital Control and Estimation - A Unified Approach. Prentice Hall, Englewood Cliffs.

Mukhopadhyay, S., A.G. Patra and G.P. Rao. 1992. "New class of discrete-time models for continuos-time systems". International Journal of Control, 55, 1161-1187.

Stericker, D.L. and N.K. Sinha. 1993. "Identification of continuous-time systems from samples of input-output data using the $\delta$-operator". Control-Theory and Advanced Technology, 9, 113-125.

Dostál, P., F. Gazdoš, V. Bobál and J. Vojtěšek. 2007. "Adaptive control of a continuous stirred tank reactor by two feedback controllers". In: 9th IFAC Workshop Adaptation and Learning in Control and Signal Processing ALCOSP'2007, Saint Petersburg, Russia, P5-1 - P5-6.

Kučera, V. 1993. "Diophantine equations in control - A survey". Automatica, 29, 1361-1375.

Bobál, V., J. Böhm, J. Fessl, and J. Macháček. 2005. Digital Self-tuning Controllers. Springer Verlag, Berlin..

\section{AUTHOR BIOGRAPHIES}

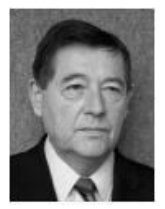

PETR DOSTÁL studied at the Technical University of Pardubice, where he obtained his master degree in 1968 and $\mathrm{PhD}$. degree in Technical Cybernetics in 1979. In the year 2000 he became professor in Process Control. He is now Professor in the Department of Process Control, Faculty of Applied Informatics of the Tomas Bata University in Zlín. His research interest are modeling and simulation of continuous-time chemical processes, polynomial methods, optimal and adaptive control.

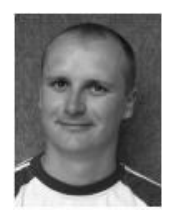

JIŘÍ VOJTĚŠEK studied at the Tomas Bata University in Zlin, where he got his master degree in chemical and process engineering in 2002 and finished his Ph.D. in Technical Cybernetics in 2007. He works as a senior lecturer in the Department of Process Control, Faculty of Applied Informatics, Tomas Bata University in Zlin.

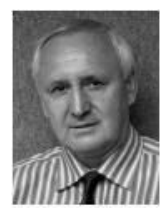

VLADIMÍR BOBÁL was born in Slavičín, Czech Republic. He graduated in 1966 from the Brno University of Technology. He received his Ph.D. degree in Technical Cybernetics at Institute of Technical Cybernetics, Slovak Academy of Sciences, Bratislava, Slovak Republic. He is now Professor in the Department of Process Control, Faculty of Applied Informatics of the Tomas Bata University in Zlín. His research interests are adaptive control systems, system identification and $\mathrm{CAD}$ for self-tuning controllers. 